MODELING, IDENTIFICATION AND CONTROL. 1983, vOL. 4, NO. 4, 195-216

doi:10.4173/mic.1983.4.1

\title{
Composition control of binary distillation columns using multivariable optimal control
}

\author{
BJARNE A. FOSS $\dagger$
}

Keywords: binary distillation columns, modeling, dual composition control, optimal control.

This article discusses dual composition control of binary distillation columns subjected to feed disturbances. A simulation model is established and some of its characteristics are discussed.

Multivariable optimal control is compared with PI-control, i.e. a SISOstructure. Simplifications of the optimal control structure are also discussed.

\section{Introduction}

A binary distillation column separates a mixture of two chemical components into two product streams. The simultaneous composition control of both these streams (dual composition control) is regarded as a difficult control problem. The main reasons for this are the transport delays and the interaction that characterizes the control loops.

The purpose of this paper is to discuss the possibilities of using the LQ-concept, i.e. optimal control based on a linear-quadratic criteria, for dual composition control. Apart from the presentation of a non-linear model for distillation processes, the investigation can be divided into two parts. Firstly, it will be established whether optimal control offers an advantage compared to conventional PI-control. Secondly, simplifications of the LQ-control structure will be investigated. Robustness of the proposed control strategies will also be an issue in the discussion.

This study is based on computer simulations of a distillation column. A model can never copy nature exactly, but the most important dynamics of many distillation processes are reproduced by the fairly simple non-linear model which will be derived.

A binary distillation unit is shown in Fig. 1. It can be divided into three parts, the column, the reboiler and the condenser and accumulator. Inside the column there are a number of plates (or stages), and the separation process is best understood by studying the relationship between vapour and liquid on each plate. A simplified explanation goes like this: On each plate the liquid and vapour holdup tries to establish a liquid-vapour equilibrium. Normally a state of equilibrium gives a higher percentage of the light component, i.e. the component with the lowest boiling point, in the vapour than in the liquid holdup. Since there is a continuous liquid flow downwards and continuous vapour flow upwards in the column, the light component tends to leave the column at the top end, while the heavy component tends to leave the column at the bottom end. The vapour flow which leaves the column at the top end is condensed in the condenser before entering the accumulator. Some of this liquid is fed back into the column as reflux. The reflux ratio (reflux flow rate to distillate flow

Received May 151983.

$\dagger$ Division of Automatic Control, The Foundation of Scientific and Industrial Research at the Norwegian Institute of Technology (SINTEF), N-7034 Trondheim-NTH, Norway. 


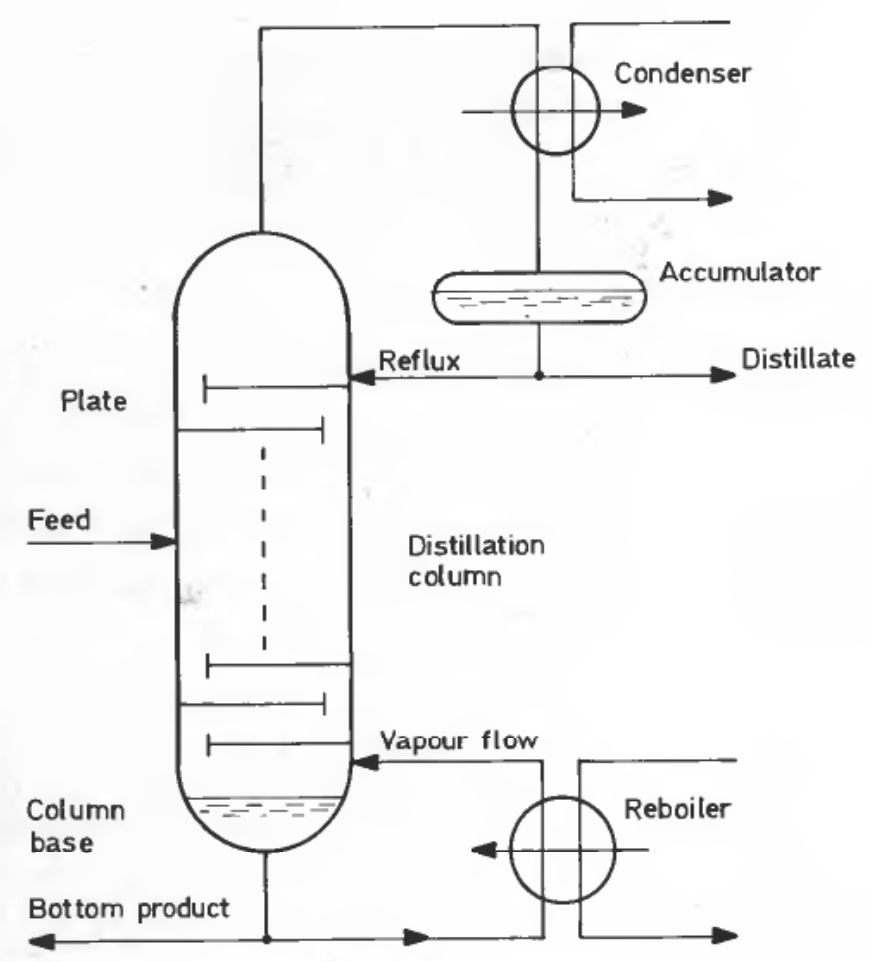

Figure 1. Binary distillation unit.

rate) is a measure of the energy input into the system. A higher ratio gives better separation as the mean mixing time in the column increases. A part of the liquid flow which leaves the bottom end of the column is vaporized in the reboiler. The ratio between the vapour flow rate and the bottom product flow rate is dependent on the reflux ratio mentioned above. This is due to the fact that an overall mass balance has to be satisfied. The feed stream normally enters a column somewhere in the middle.

The total number of control inputs is five-cooling rate (condenser), reflux flow rate, distillate flow rate, heat input (reboiler) and bottom product flow rate. A normal operating requirement is constant pressure in the column. The pressure is usually controlled by the cooling rate. Two variables are used to control the liquid holdup in the accumulator and in the column base. In the simulation experiments in this paper the accumulator holdup is controlled by the distillate flow rate while the base holdup is controlled by the bottom product flow rate. This leaves only the reflux flow rate and the heat input for composition control.

The selected configuration is a typical one in processes with modest reflux ratios $(<5)$ (Luyben 1979). The distillate flow rate is normally too small for accumulator holdup control for higher ratios. In these cases the reflux flow rate is used instead of the distillate flow rate.

Dual composition control is the exception rather than the rule in industrial plants. There are several reasons for this. Firstly, a column often operates with a constraint on a control variable most of the time, e.g. maximum heat input. This reduces the degrees of freedom in the process, and dual composition control is not possible. Secondly, there has been a widespread opinion that the added complexity of dual composition control does not pay off. Higher energy prices have influenced this 
opinion since distillation processes may consume as much as $40 \%$ of the total energy used in a petrochemical plant (Martin et al. 1981). The energy saving potential of dual composition control can be seen from the following argument. If one input variable is kept constant and a producer still wants both product streams to satisfy certain composition requirements, the fixed variable must be set according to the worst possible case that may arise. Hence, the energy input under average conditions is too high. Thirdly, reluctance to the use of dual composition control in real life is influenced by the difficulty of solving the control problem in a satisfactory way.

Papers discussing the dual composition control problem on a multivariable LQbasis generally conclude that substantial improvements can be achieved compared with a single loop control strategy (Hu and Ramirez 1972, Gilles and Retzbach 1980, Hammarstrom 1980 and Eckelmann 1980). The bulk of these papers are, however, based on simulation results, or at most, on pilot column experiments. Practical tests on production plants are rare, and encouraging simulation results may be difficult to copy in real life. All the same, a critical view of simulation results, i.e. taking into account both the inadequacies of a process model and the measurement problems which arise in real life, can tell something about the capabilities of different control systems.

\section{The model}

This section gives a description of the non-linear model which is used for the control study in this article. The dynamic equations for a plate distillation process can be established by writing down the mass and thermal balances on each plate in a column. Simplifications have to be introduced in order to make the problem tractable. The extent to which the model is simplified strongly depends on the use of the model.

\subsection{Basic assumptions}

Figure 2 gives a detailed picture of the holdups and streams around a plate in a binary distillation column. Liquid flow $\left(L_{i+1}\right)$ enters the plate in the downcomer and then passes $\left(L_{i+1}^{\prime}\right)$ on to the actual plate. Vapour flow $\left(V_{i-1}\right)$ penetrates the liquid

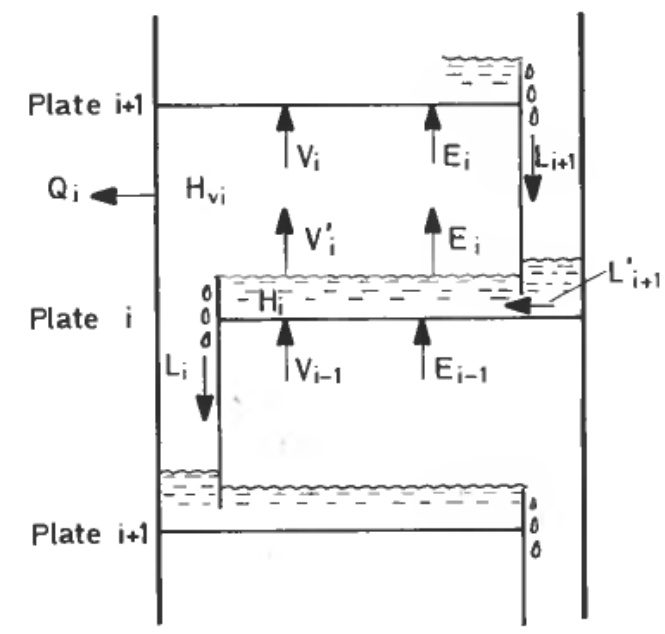

Figure 2. Description of the flows and holdups on a plate. 
holdup $\left(H_{i}\right)$ from below and interacts with it in order to establish a vapour-liquid equilibrium. When the vapour flow passes through the liquid holdup, some of the liquid $\left(E_{i}\right)$ will follow the vapour flow. This is called interstage backmixing. Along with the fact that liquid-vapour equilibrium on each plate is unobtainable, it is the major reason why distillation units ate prevented from operating like countercurrent equilibrium processes (Loud and Waggoner 1978). $Q_{i}$ indicates a loss of thermal energy on a plate.

The equations governing steady-state relationships are to a great extent, empirical and are quite complex, for example, the liquid overflow $L_{i}$. It is dependent on the liquid height above the outlet weir. This height, however, cannot be calculated directly since the liquid holdup consists mainly of froth and foam.

This rather short description shows the complexity of models based on a detailed description of the behaviour on each plate. Fortunately, several simplifications can be made for the purpose of obtaining a model relevant to a wide range of distillation processes, which can be used for control studies. The assumptions will be discussed in the following.

(a) Vapour holdup is negligible compared to liquid holdup $\left(H_{v, i}=0\right)$. This assumption makes it possible to eliminate the composition dynamics of the vapour phase. As a rule it can be justified for all but high pressure systems (see Stainthorp and Searson 1973). In addition to omitting the vapour phase dynamics, constant pressure is assumed throughout the column.

(b) Well mixed liquid stage. The liquid in the downcomer and on the plate are not separated, and no concentration gradients are accounted for in the liquid holdup. These simplifications normally have small effects on the dynamic behaviour of the column.

(c) No interstage backmixing. This restriction can be disputed for columns with high vapour flows since the entraintment $\left(E_{l}\right)$ increases with increased vapour flow (Loud and Waggoner 1978).

(d) No change of thermal energy and negligible exchange of enthalpy between liquid and vapour streams on a plate.

These simplifications may appear controversial. For binary mixtures where the molar latent heats of vaporization of the two components are quite similar, the vapour flow will only exchange very little thermal energy with the liquid on a plate. Assuming small heat losses from the column disregards the need for an enthalpy balance equation on each plate. In most cases this leaves us with a model that is adequate for preliminary control studies (see Stainthorp and Searson 1973 and Levy et al. 1969).

\subsection{A non-linear simulation model}

A 21-plate column model will be derived. The restrictions $(a)-(d)$ imposed above leave two state variables on each plate. These are chosen as the mole fraction of the light component in the liquid phase (in the following named the composition) and the liquid holdup in moles. The plate numbers increases upwards in the column.

As mentioned in the introduction, the control structure keeps a constant liquid holdup in the column base. Only one state variable is therefore necessary to characterize the column base/reboiler. A minor detail should be mentioned. The model 
measures the holdup in moles. Hence, a level control for the base level must be related to the composition in the base. This also applies to the accumulator.

One state variable, the composition in the condenser/accumulator is sufficient to characterize the top section.

It is assumed that the reflux is at its boiling point when returning to the column. This is no severe restriction as any other assumption can be met by adding a heatbalance equation around the condenser/accumulator and top-plate.

The feed stream is introduced on the middle plate (plate no. 11) of the column. It is assumed to be liquid. The amount of vapour which is condensed is negligible if the feed stream is fairly near its boiling point because the heat of vaporization always will be much larger than the heat capacity of the liquid mixture.

The main symbols of this subchapter are:

$B$ Bottom product flow rate $(\mathrm{mol} / \mathrm{s})$

$D$ Distillate flow rate $(\mathrm{mol} / \mathrm{s})$

$H_{i} \quad$ Liquid holdup (mol)

$L_{i} \quad$ Liquid flow rate $(\mathrm{mol} / \mathrm{s})$

$R \quad$ Reflux flow rate $(\mathrm{mol} / \mathrm{s})$

$V$ Vapour flow rate $(\mathrm{mol} / \mathrm{s})$

$x_{i} \quad$ Liquid composition (i.e. mole fraction of light component)

$x_{F} \quad$ Feed composition

$y_{i} \quad$ Vapour composition

A process model may now be established.

The state equations for a plate in the column (not the feed plate).

$$
\begin{aligned}
\left(\dot{H}_{i} x_{i}\right) & =L_{t+1} x_{i+1}+V y_{i-1}-L_{i} x_{i}-V y_{i} \\
\dot{H}_{i} & =L_{t+1}-L_{i}
\end{aligned}
$$

Equation (1) may be simplified.

$$
H_{i} \dot{x}_{i}=L_{i+1}\left(x_{i+1}-x_{i}\right)+V\left(y_{i-1}-y_{i}\right)
$$

The dynamic equations now become,

Column base/reboiler

$$
H_{1} \dot{x}_{1}=L_{2}\left(x_{2}-x_{1}\right)+V\left(x_{1}-y_{1}\right)
$$

Column plate, except for the feed plate

$$
\begin{array}{rlrl}
H_{i} \dot{x}_{i} & =L_{i+1}\left(x_{i+1}-x_{i}\right)+V\left(y_{i-1}-y_{i}\right), & i=2, \ldots, 11,13, \ldots, 22 \\
\dot{H}_{i} & =L_{i+1}-L_{i}, & i & =2, \ldots, 11,13, \ldots, 22
\end{array}
$$

Feed plate

$$
\begin{aligned}
H_{12} \dot{x}_{12} & =L_{13}\left(x_{13}-x_{12}\right)+V\left(y_{11}-y_{12}\right)+F\left(x_{F}-x_{12}\right) \\
\dot{H}_{12} & =L_{13}-L_{12}+F
\end{aligned}
$$

Condenser/Accumulator

$$
H_{23} \dot{x}_{23}=V\left(y_{22}-x_{23}\right)
$$


Since it may seem a little confusing, it should be emphasized that the plate numbers $1,2, \ldots, 21$ upwards in the column correspond to the state variables $2,3 \ldots, 22$.

The product flows can be calculated as constant holdup is assumed in the column base $\left(H_{1}\right)$ and in the accumulator $\left(H_{23}\right)$.

$$
\begin{aligned}
& B=L_{2}-V \\
& D=V-R
\end{aligned}
$$

It is easy to verify an overall material balance for static conditions since the liquid flow rate in the stripping section (plates below the feed plate) equals the sum of the feed flow rate and the reflux flow rate.

$$
B+D=\left(L_{2}-V\right)+(V-R)=L_{2}-R=F
$$

Equations for the vapour concentration $\left(y_{i}\right)$ and the liquid overflow $\left(L_{i}\right)$ remain to be found.

The Francis weir formula relates the plate holdup to the liquid overflow.

$$
L_{i}=1 \cdot 707 \frac{\rho_{i}}{M_{l}} l_{w}\left(h_{i}-h_{w}\right)^{3 / 2}
$$

$\rho_{i}$ denotes the liquid density, $M_{i}$ the molweight, $l_{w}$ the length of the outlet weir, $h_{w}$ the height of the outlet weir and $h_{i}$ the liquid height on plate $(i-1)$. The liquid height is difficult to obtain since foam and froth is present on every plate.

The following relationship is used

$$
h_{i}=\frac{h_{i}^{0}}{\beta}
$$

$h_{i}{ }^{\circ}$ liquid height without any formation of foam and froth

$\beta$ the froth to liquid density ratio

The $\beta$-factor may vary, it is particularly sensitive to changes in the vapour flow changes.

A common way of relating the vapour and liquid concentration is through the relative volatility, $\alpha$. In many cases it gives a good approximation for the constant pressure case.

$$
y=\frac{\alpha x_{i}}{1-(\alpha-1) x_{i}}, \alpha>1
$$

Distillation columns cannot obtain a liquid-vapour equilibrium on each plate during operation. Murphree's tray liquid efficiency is a common way to relate the real vapour composition to the idealized composition given in eqn. (15). For this investigation the tray efficiency has been set equal to one which means that the idealized vapour composition is taken as the real one. This is often done in control studies of fairly simple distillation models, see e.g. Hu and Ramirez (1972). The parameters used in the model are shown in Table 1. 


\begin{tabular}{lc}
\hline \multicolumn{1}{c}{ Parameter } & Value \\
\hline Holdup in column base/reboiler & $500 \cdot 0 \mathrm{~mol}$ \\
Holdup in accumulator/condenser & $250 \cdot 0 \mathrm{~mol}$ \\
Weir length (eqn. (13)) & $0 \cdot 18 \mathrm{~m}$ \\
Weir height (eqn. (13)) & $0 \cdot 15 \mathrm{~m}$ \\
Plate area & $0 \cdot 12 \mathrm{~m}^{2}$ \\
$\beta$-factor (eqn. (14)) & $0 \cdot 50$ \\
Relative volatility (eqn. (15)) & $1 \cdot 30$ \\
Density of light component & $879 \mathrm{~kg} / \mathrm{m}^{3}$ \\
Density of heavy component & $866 \mathrm{~kg} / \mathrm{m}^{3}$ \\
Molweight of light component & $78 \mathrm{~kg} / \mathrm{kmol}$ \\
Molweight of heavy component & $92 \mathrm{~kg} / \mathrm{kmol}$ \\
\hline
\end{tabular}

Table 1. Parameter values for the non-linear model.

\begin{tabular}{lc}
\hline \multicolumn{1}{c}{ State variable } & Stationary value \\
\hline Composition of the bottom product & $28 \cdot 24 \%$ \\
Composition on plate no. 1 & $32 \cdot 60 \%$ \\
Composition on plate no. 6 & $45 \cdot 52 \%$ \\
Composition on feed plate no. 11 & $49 \cdot 57 \%$ \\
Composition on plate no. 6 & $52 \cdot 57 \%$ \\
Composition on plate no. 21 & $66 \cdot 12 \%$ \\
Composition of the distillate & $71 \cdot 73 \%$ \\
Holdup on plate no. 1 & $98 \cdot 19 \mathrm{~mol}$ \\
Holdup on plate no. 6 & $100 \cdot 34 \mathrm{~mol}$ \\
Holdup on feedplate & $101 \cdot 03 \mathrm{~mol}$ \\
Holdup on plate no. 16 & $98 \cdot 99 \mathrm{~mol}$ \\
Holdup on plate no. 21 & $101 \cdot 38 \mathrm{~mol}$ \\
\hline
\end{tabular}

Table 2. Stationary values for some of the state variables subjected to the conditions outlined in eqn. (16) and Table 1.

The stationary values of the feed flow rate/composition, the reflux flowrate and vapour flowrate are set equal to

$$
\begin{aligned}
& \text { Feed composition } x_{F}=50 \%(0.5 \text { mol-fraction of light component }) \\
& \text { Feed flow } F=2.0 \mathrm{~mol} / \mathrm{s}(\simeq 0.2 \mathrm{l} / \mathrm{s}) \\
& \text { Reflux flow rate } \quad R=2.5 \mathrm{~mol} / \mathrm{s} \\
& \text { Vapour flow rate } \quad V=3.5 \mathrm{~mol} / \mathrm{s}
\end{aligned}
$$

The product streams are both $1 \cdot 0 \mathrm{~mol} / \mathrm{s}$.

A few comments should be made in advance on the presentation of the simulation experiments. All curves regarding composition changes view the mole fraction of the light component in percentages, i.e. the values of the composition state variables multiplied by 100 . 
PLATE NUMBER

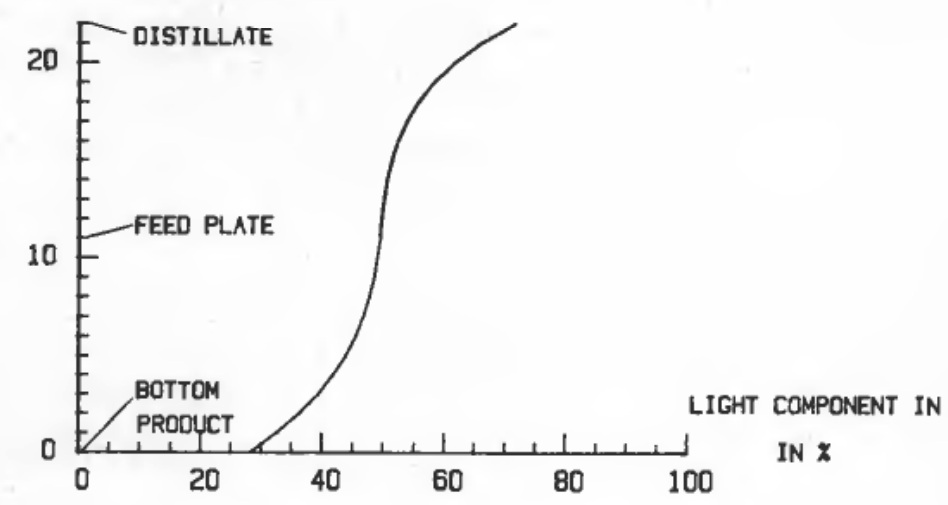

Figure 3. Steady-state composition profile subjecting the non-linear model to the conditions outlined in eqn. (16) and Table 1.

The stationary values of some of the state variables subjected to the conditions outlined in eqn. (16) and Table 1 are shown in Table 2. The composition profile can be viewed in Fig. 3.

The dynamics of the non-linear model are governed by the hydraulic and composition modes. These are quite easily separated since the dynamics of the hydraulic modes are much faster than the composition modes. Figure 4 illustrates this. At time zero the process is excited by a step change in the feed flow rate $(2 \cdot 0$ to $2.4 \mathrm{~mol} / \mathrm{s})$. The first $15 \mathrm{~s}$ the molar holdup increases rapidly due to the higher liquid flow which penetrates down through the stripping section of the column. Later the holdup changes slowly as the composition increases.

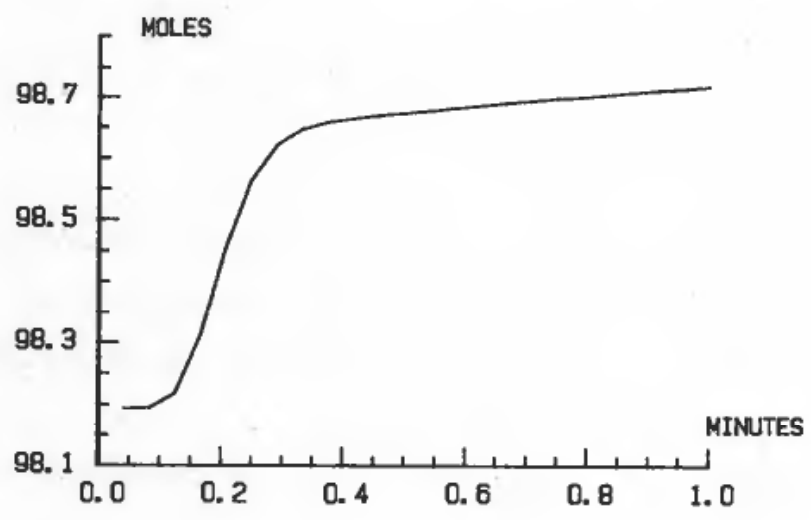

Figure 4. Liquid holdup on the bottom plate (plate no. 1) in response to a step change in the feed flow rate at time zero.

The stiffness ratio of a model is defined as the ratio between its largest and smallest eigenvalue. Models of distillation columns are generally quite stiff, this is illustrated by Fig. 4 . The fast holdup response $(t<0.4 \mathrm{~min})$ is caused by the time constants of the hydraulic modes while the dominant composition modes give a 
slowly rising liquid holdup $(t>0 \cdot 4 \mathrm{~min})$ due to composition changes. Luyben et al. (1975) discusses the phenomenon and one of the conclusions are that improved separation (higher reflux ratio) increases the stiffness of a model. Looking at the eigenvalues of a linearized model when neglecting the hydraulic models, supports this conclusion.

The stationary values of Table 2 gives a stiffness ratio of 160 . Increasing the reflux ratio from $2 \cdot 5$ to $5 \cdot 0$, increases the stiffness ratio to 390 . The stiffness ratio of the total model is considerably higher because of the hydraulic modes.

Numerical stability while simulating stiff models is a problem. In this investigation a fairly simple integration algorithm (fourth order Runge-Kutta) with a short timestep is used to secure numerical stability.

Figure 5 shows the product compositions after a step change in the reflux flow rate. The responses are not very exciting, but they give a feel for the dynamics of a column. Another important fact is the strong interaction between the reflux and the bottom product composition. Figure 5 also shows similar responses to a step change in the vapour flow rate.

The same simulation for a higher reflux ratio gave slower responses to step changes in both the reflux and vapour flow. Intuitively this stands to reason as the

(a)

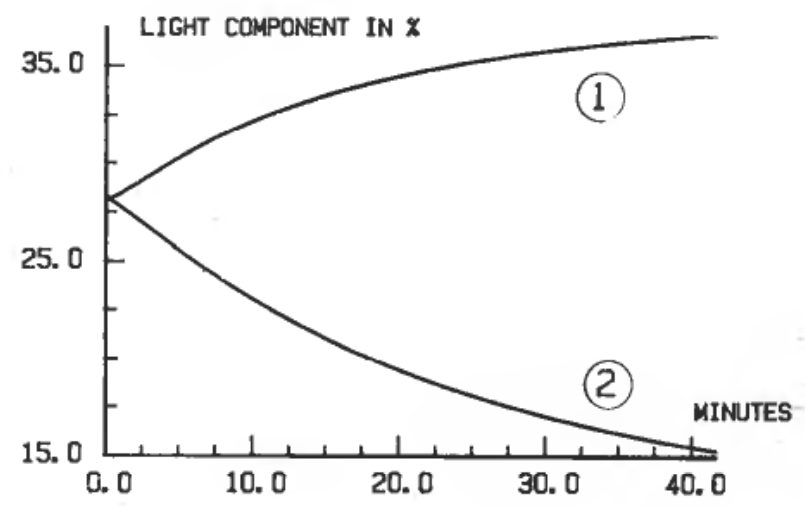

(b)

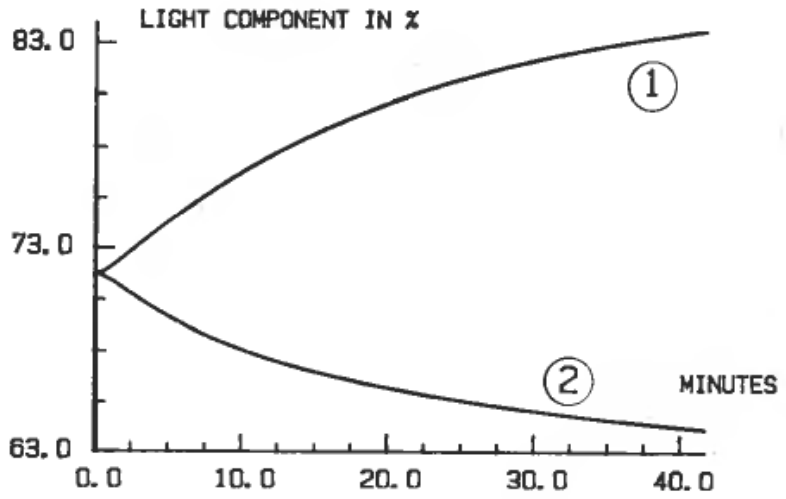

Figure 5. Composition responses to a step change in the reflux flow rate (1) and the vapour flow rate (2) at time zero for (a) bottom product composition and (b) distillate composition. 
mean mixing-time in the column increases. The model dynamics are not affected to any great extent by changes in the relative volatility. It will, however, indirectly influence the dynamics of the control loops since a change in the relative volatility imposes a change in the reflux ratio in order to maintain constant product quality.

Viewing the inputs, the reflux dynamics are very fast and will therefore be neglected. The boiler's dynamics cannot be treated in the same way. Its maximum capacity must be approximately $200 \mathrm{~kW}$ in order to satisfy the required boiling rates in the simulations. A reasonable model seems to be

$$
\dot{V}=-\frac{1}{T_{B}}\left(V-V^{\prime}\right)
$$

$T_{B}$ boiler time constant, chosen to be $1 \cdot 0 \mathrm{~min}$

$V^{\prime}$ demanded vapour flow rate

$V$ actual vapour flow rate

\section{Dual composition control}

In this section multivariable optimal control will be compared with conventional PI-control, i.e. a SISO-structure. Simplifications of the LQ-control structure will also be considered. The controllers will be subjected to feed flow/feed composition disturbances, and evaluated according to their ability to compensate for these disturbances. As mentioned earlier the reflux flow rate and the vapour flow rate (heat input) are used as control inputs for composition control.

A brief discussion of the LQ-concept related to the specific use in a distillation process is given below. Only continuous control will be considered, i.e. problems specially associated with discrete time measurements/control signals are neglected.

The linearized model which is the basis for calculating the control law, assumes constant liquid holdup on each stage. There are two reasons for this. Firstly, the controller is used for composition control. The hydraulic modes associated with the liquid holdup are considerably faster than the composition modes. Constant liquid holdup can therefore be assumed without losing any significant amount of control performance. Secondly, the complexity of the control law is considerably reduced by omitting the hydraulic modes. The state vector of the linearized model consists of the composition on each plate including the composition of the product streams.

Linearized model

$$
\left.\begin{array}{rl}
\dot{x} & =A x+B u \\
a_{i j} & =\partial f_{i} / \partial x_{i} \\
b_{i 1} & =\partial f_{i} / \partial V \\
b_{i 2} & =\partial f_{i} / \partial R
\end{array}\right\} \text { (calculated using stationary state-values) }
$$

$f_{i}$ is given by eqns. (4), (5), (7), and (9). The control signal $u$ is composed of the vapour flow rate $V$, and the reflux flow rate $R$.

The LQ-controller minimizes

$$
J=\frac{1}{2} x^{T}\left(t_{2}\right) S x\left(t_{2}\right)+\frac{1}{2} \int_{t_{1}}^{t_{2}}\left(x^{T}(t) Q x(t)+u^{T}(t) P u(t)\right) d t
$$


In the criteria it is assumed that $P$ is positive definite while $Q$ is positive semi-definite. The solution is found by solving the dynamic Riccati equation. The resulting feedbacklaw is time-varying. If the optimization interval is infinitely long the feedback matrix becomes time-invariant (this assumes constant $A$ and $B$ matrices in eqn. (18)). In practice this means that the optimization interval is considerably longer than any of the system dynamics of eqn. (18). The algebraic Riccati equation can be solved in order to find the feedback matrix $G$.

$$
\begin{gathered}
R A+A^{T} R-R B P^{-1} B^{T} R+Q=0 \\
G=-P^{-1} B^{T} R
\end{gathered}
$$

Since steady-state changes in the feed flow/feed concentrations will appear, integral action must be included in the controller. This is done by augmenting the state vector of eqn. (18) with the integrated product stream deviations.

The augmented state vector also incorporates the actual vapour flow, confer eqn. (17). This is partly based on the experience in Foss (1980). This reference underlines the difficulty of getting satisfactory control performance when boiler dynamics are excluded in the linearized model from which the optimal control law is calculated.

$$
\left.\begin{array}{l}
\tilde{x}^{T}=\left[x^{T}, z_{1}, z_{2}, V\right] \\
\dot{z}_{1}=x_{1}-x_{1 \mathrm{ref}} \\
\dot{z}_{2}=x_{23}-x_{23 \mathrm{ref}}
\end{array}\right\}
$$

The augmented model now becomes

$$
\dot{\tilde{\boldsymbol{x}}}=\tilde{A} \tilde{\boldsymbol{x}}+\tilde{B} \boldsymbol{u}
$$

The weight matrix $Q$ is also augmented

$$
\tilde{Q}=\left[\begin{array}{lll}
Q_{1} & 0 & 0 \\
0 & Q_{2} & 0 \\
0 & 0 & Q_{3}
\end{array}\right]
$$

$Q_{1}$ weights the compositions, $Q_{2}$ the two integrated product composition deviations and $Q_{3}$ the vapour flow.

There is no easy way of finding weight matrices that give good control. The structure of distillation columns can, however, be of some help. Intuitively it seems right to weight deviations in the compositions near the bottom and top end of the column the most, since it is the product streams' deviation from their reference values that are the prime interest of the controller.

The $Q_{1}$-matrix of eqn. (24) is therefore chosen as

$$
Q_{1}=\operatorname{diag}(1 \cdot 0,0 \cdot 1,0 \cdot 0, \ldots, 0 \cdot 0,0 \cdot 1,1 \cdot 0]
$$

The $P, Q_{2}$ and $Q_{3}$-weight matrices were assumed to have the following structure

$$
P=\epsilon_{1} I \quad Q_{2}=\epsilon_{2} I \quad Q_{3}=\epsilon_{3}
$$

where $I$ denotes the identity matrix. 
The problem of finding $P$ and $Q$ is now reduced considerably. Weight matrices that give good control were fairly easy to find. The computed feédback gain matrix shown in Table 3 gave excellent performance of the closed-loop system. It is used in the simulation run shown (as $L Q$ ) in Figs. $6(a)$ and $(b)$. The excitation of the process

$$
\begin{aligned}
& {\left[\begin{array}{rrrrrrrr}
399 \cdot 2 & 53.0 & 3.4 & 21.7 & 12.97 & 7.33 & 3.84 & 1.81
\end{array}\right.} \\
& {\left[\begin{array}{llllllll}
-47.1 & -1.9 & 1.1 & 1.8 & 1.51 & 1.00 & 0.54 & 0.23
\end{array}\right.} \\
& \begin{array}{llllllll}
0.71 & 0.18 & 0.00 & 0.00 & 0.04 & -0.11 & -0.66 & -1.90
\end{array} \\
& \begin{array}{llllllll}
0.05 & -0.02 & -0.03 & 0.00 & 0.06 & 0.10 & 0.09 & -0.05
\end{array} \\
& \begin{array}{rrrrrrr}
-4 \cdot 29 & -8 \cdot 46 & -15 \cdot 27 & -25 \cdot 7 & -40 \cdot 8 & -61 \cdot 5 & -215 \cdot 8 \\
-0.50 & 1.66 & -4 \cdot 41 & -10 \cdot 5 & -23 \cdot 1 & -47 \cdot 7 & -224 \cdot 5
\end{array} \\
& \left.\begin{array}{rr|r}
4 \cdot 47 & -2 \cdot 24 & -1 \cdot 58 \\
-2 \cdot 24 & -4 \cdot 47 & 0.53
\end{array}\right]
\end{aligned}
$$

Table 3. Feedback matrix for $\epsilon_{1}=0 \cdot 01, \epsilon_{2}=0 \cdot 25$ and $\epsilon_{3}=0 \cdot 00$.

(a)

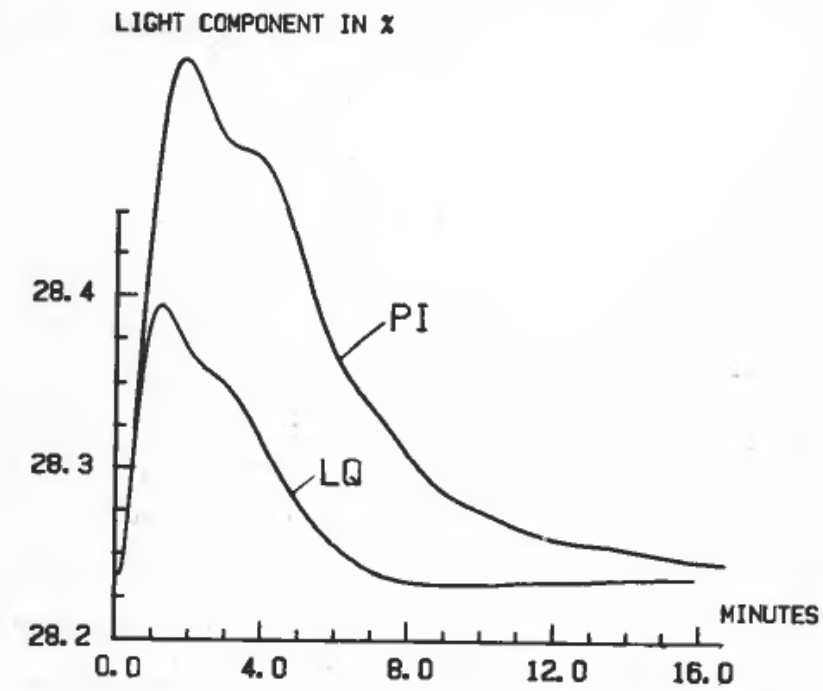

(b)

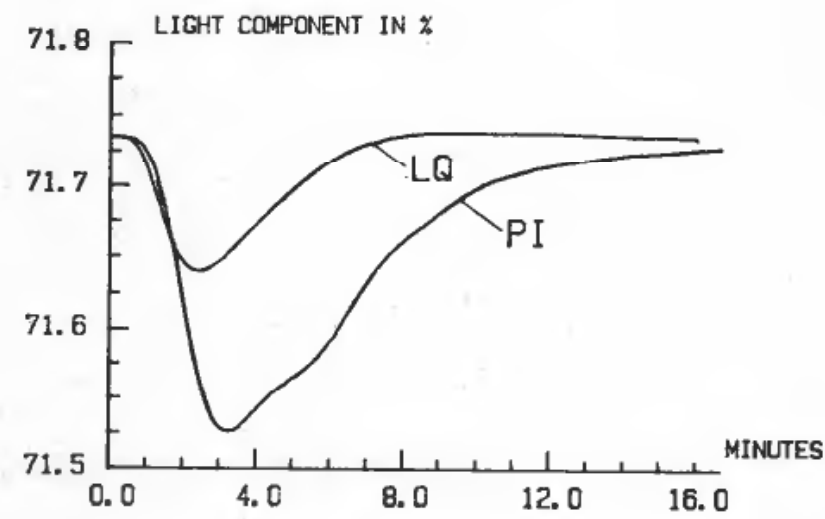


(c)

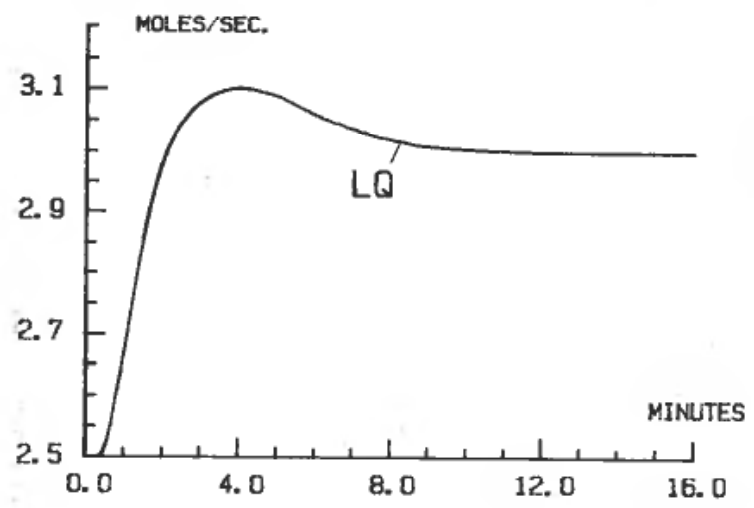

(d)

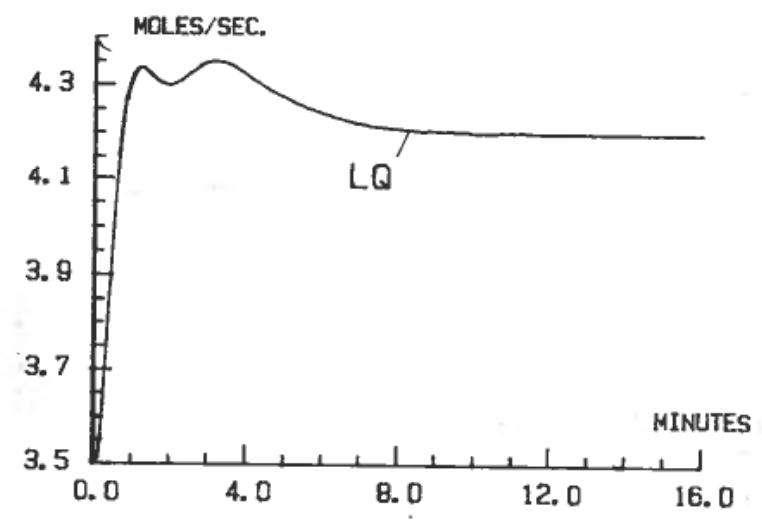

Figure 6. Responses to a step change in the feed flow rate using a LQ-controller (LQ) or PI-controllers (PI) for (a) bottom product composition, (b) distilled composition, (c) reflux flow rate and $(d)$ vapour flow rate.

is a step change in the feed flow rate $(2.0$ to $2.4 \mathrm{~mol} / \mathrm{s})$ at time zero. The controller compensates neatly for the disturbance. The control signals can be viewed in Figs. $6(c)$ and $(d)$. Changes in the feed composition are compensated equally well.

It should be emphasized that the reference values for the product compositions are equal to the initial values (time zero) for these compositions. This will be the case in all the following simulation experiments. This is because a discussion on the different controllers' ability to follow reference changes will be omitted. The simulations also assume no measurement time-delay.

Since the reboiler dynamics are included in the linearized model, the actual vapour flow rate should be measured. In general it is not easy to obtain a good measurement of this vapour flow rate. If however, the measurement contains too much noise, an estimation algorithm based on a simple model of the reboiler can produce a better estimate of the vapour flow than a noisy measurement.

The multivariable controller is compared with a multiple SISO control, i.e. using two PI-controllers to control respectively the distillate through the reflux and the bottom product through the vapour-flow rate. They were tuned simultaneously to reasonable performance. Subjected to the same disturbance as the LQ-controller above, the controllers gave a response as shown in Figs. $6(a)$ and $(b)$ (PI). As could be expected these controllers cannot compete with the multivariable structure. 
The LQ-controller discussed above requires measurements of the composition on every stage in the column in addition to the product streams and the vapour-flow. It is important to investigate how the LQ-controller can be simplified without losing its superiority versus the conventional controller. Many of the elements in the feedback matrix in Table 3 are small. Viewing the size of the elements can, however, give a somewhat false picture of their relative importance. One has to bear in mind that the composition deviations are much larger in the middle of the column than at the top and bottom end, when the column is subjected to disturbances in the feed.

This discussion indicates that an approach based on feedback from a reduced state-vector could be successful. In Foss (1980) the reduced feedback gain matrix was calculated using two different methods. One of them implied solving a Liapunovequation, while the other simply used the matrix elements from the full feedback matrix for the states that were measured. There were no great differences in the two methods, only the simplest one will therefore be implemented here.

Simulations on a seven stage column without boiler dynamics (Foss 1980) showed that the number of states in the feedback loop could be reduced considerably while still maintaining tight control. Measurements of the product compositions were necessary to maintain integral action in the controller. Only two measurements gave an unstable system whereas a third measurement in the controller made it remarkably well behaved. The location of this third measurement was however, quite critical. The optimal location was at the top end of the column. Locating it in the middle or lower part of the column gave an unstable system. Similar results are reported in Hammarstrom (1980) for a twelve stage distillation column.

To check the validity of these results on a larger column, simulations were performed using the non-linear model of a 21-stage column. A step change in the feed flow rate $(2.0$ to $2.4 \mathrm{~mol} / \mathrm{s})$ at time zero was again used as the disturbance. Figure 7 shows the results measuring the compositions of the two product streams, the vapour flow rate and the composition on stage $20\left(x_{21}\right)$ (curve 2) or on stage $21\left(x_{22}\right)$ (curve 1). Curve 3 in Fig. $7(a)$ shows a simulation experiment where the third composition measurement has been omitted. It underlines the necessity for the third composition measurement.

The results verify that the additional measurement should be located as high up in the column as possible. The reason for the critical location of the third measurement is that the controller needs a certain amount of information about the column's dynamics to perform satisfactorily. This is obtained by locating the third measurement at the top end of the column. Compared with Fig. 6 (LQ) the system behaves remarkably well taking into account the fact that the number of composition measurements are reduced from 23 to 3 . Neglecting the vapour flow measurement from the boiler in the feedback loop was not successful. It gave an unstable controller.

Feedforward compensation in the controllers will now be discussed. This may be introduced as a part of the LQ-control structure. A simpler algorithm will however be used here. The obvious way of introducing feedforward is through a measurement of the feed flow rate. This is both unexpensive and simple. The measurement is used to manipulate the vapour flow rate. The time constant of the boiler is far longer $(60 \mathrm{~s})$ than the liquid transport delay from the feed section to the bottom-end of the column $(\approx 10 \mathrm{~s})$. This is the cause for omitting dynamics in the feedforward compensation. The gain is chosen such that the liquid to vapour flow ratio in the stripping section (lower half) of the column, stationary speaking, is maintained. This secures unchanged separation in this section. 
(a)

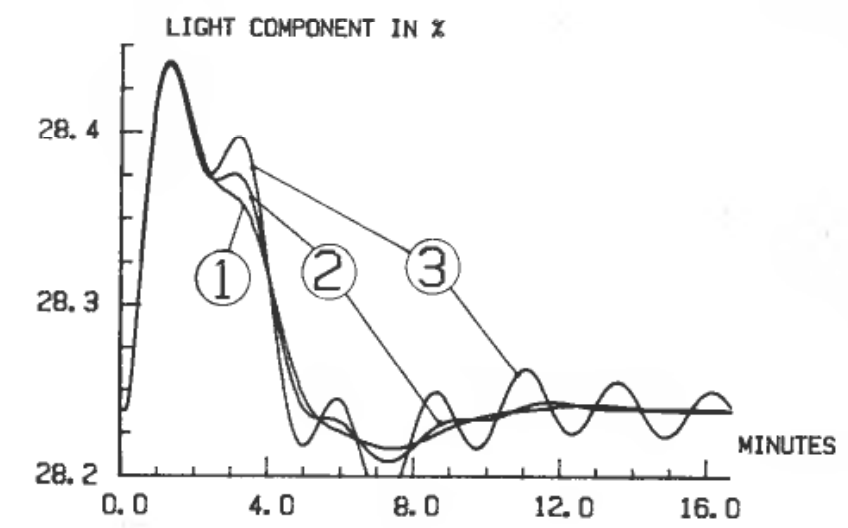

(b)

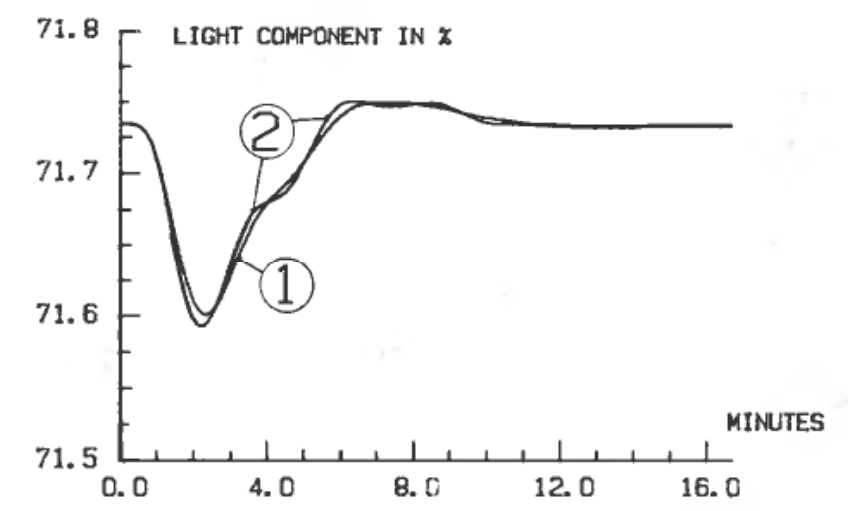

Figure 7. Responses to a step change in the feed flow rate using a LQ-control structure with a reduced state feedback for $(a)$ bottom product composition and $(b)$ distillate composition.

Feedforward compensation

$$
\Delta V=\frac{V_{\text {so }}}{L_{\text {so }}} \Delta F
$$

$V_{\mathrm{So}}$ and $L_{\mathrm{So}}$ denotes stationary vapour and liquid flow rates in the stripping section, whereas $\Delta V$ is the vapour flow rate change, and $\Delta F$ is the feed flow rate change. In the model used in this article $V_{\text {so }}$ is equal to the stationary vapour flow while $L_{\text {so }}$ equals the sum of the reflux and the feed flow rate.

Figures $8(a)$ and $(b)(\mathrm{PI})$ show the response using the two PI-controllers introduced earlier in addition to the feedforward compensation. These responses may be compared with Figs. $6(a)$ and $(b)(\mathrm{PI})$. The improvement is apparent.

In Figs. $8(a)$ and $(b)$ (LQ) the reduced state feedback (three composition measurements and a vapour flow rate measurement) control strategy is combined with feedforward compensation. These responses should be compared with Figs. $7(a)$ and $(b)$ (curve 1). The simulations show that feedforward compensation introduced at low cost give substantial improvements to the compensation of feed flow disturbances. Feedforward compensation of feed composition changes is not that easy to justify. Firstly, the additional measurement is more costly and secondly, this composition varies quite slowly. 
(a)

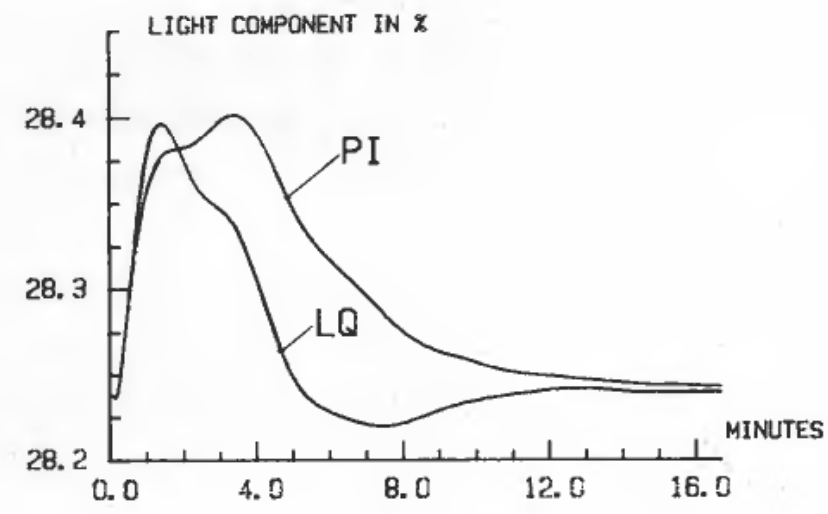

(b)

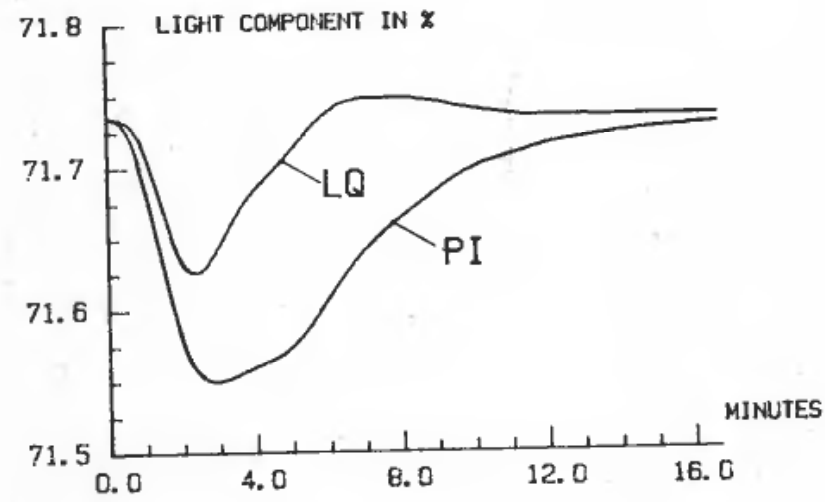

Figure 8. Responses to a step change in the feed flow rate using a LQ-control structure with reduced state feedback (LQ) or PI-controllers (PI) for (a) bottom product composition and $(b)$ distillate composition. In addition feedforward compensation is added.

Returning to the task of simplifying the feedback loop of the optimal control law. A further reduction in the number of measurements will require the use of estimation techniques. This idea will not be pursued in the case of the vapour flow measurement. Instead the possibility of omitting the third composition measured at the top end of the column will be investigated in some detail. The goal is to find a simple way of estimating this composition without sacrificing too much in control performance.

The approach which is used here is based on the fact that the composition dynamics of a column are fairly slow. This gives the reason for choosing a very simple estimator model (an integrator) for the composition on stage 21.

$$
\dot{x}_{22}=e
$$

$e$ is white noise with zero mean value.

The composition at the top end of the column $\left(x_{22}\right)$ affects the distillate composition through the dynamics of the condenser/accumulator, see eqn. (9). The estimator shown in Fig. 9 naturally emerges. $k_{1}$ and $k_{2}$ denotes the estimation gain factors. If there is no measurement time-delay, the time lag between the real and the estimated composition on stage $21\left(x_{22}\right)$ can be made arbitrary small by increasing the gain 


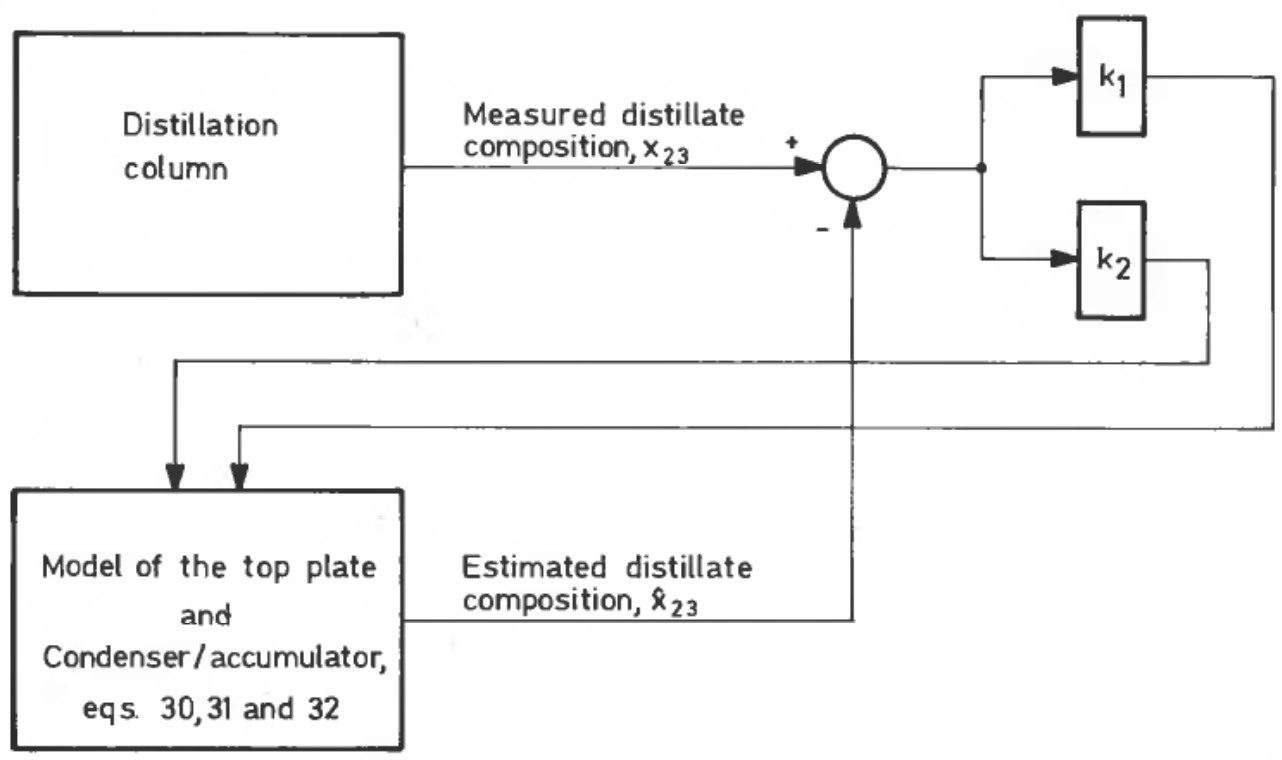

Figure 9. A simple estimation loop for the top end of a distillation unit.

factors $k_{1}$ and $k_{2}$. In practice the gain factors are limited by the measurement noise of the top product composition measurement.

The factors are calculated using the stationary Kalman-filter equations. The stationary values in Table 2 are used to compute the system matrix of the estimation model while the process noise matrix is chosen as

$$
V_{N}=\left[\begin{array}{cc}
10^{-2} & 0 \\
0 & 10^{-4}
\end{array}\right]
$$

It seems reasonable to assume more noise excitation on the composition estimate on stage 21 than on the composition estimate of the distillate since the former statevariable is modeled very crudely. By choosing a measurement variance of $W=0 \cdot 10$ the following $K$-vector is obtained

$$
K=\left[\begin{array}{l}
k_{1} \\
k_{2}
\end{array}\right]=\left[\begin{array}{l}
0.320 \\
0.082
\end{array}\right]
$$

The equations of the estimation loop are

$$
\begin{aligned}
\hat{x}_{22} & =k_{1} \epsilon \\
\dot{\hat{x}}_{23} & =\frac{V}{H}\left(\hat{y}_{22}-\hat{x}_{23}\right)+k_{2} \epsilon \\
\epsilon & =x_{23}-\hat{x}_{23}
\end{aligned}
$$

$\wedge$ denotes estimated values. $\hat{y}_{22}$ is calculated using $\hat{x}_{22}$ in eqn. (15). The estimate of $x_{22}$ is obtained through an integrator, while eqn. (31) gives the estimate of the top product composition after filtering $\hat{y}_{22}$ through the dynamics of the accumulator. 
A simulation experiment with the same feed disturbance, the same feedback gains and the same feed forward composition as in Figs. $8(a)$ and $(b)(\mathrm{LQ})$ was run. The only difference was that the estimated composition on stage 21 replaced the true value. The result is shown in Figs. $10(a)$ and $(b)$ (curve 1) while Fig. $10(c)$ compares the estimated composition on stage 21 with the true value.

If the estimation-loop is made slower, i.e. decreasing the values in the $K$-vector, the responses tend to become oscillatory. This is shown, although not very pronounced, in Figs. $10(a)$ and $(b)$ (curve 2) using the following $K$-vector

$$
K=\left[\begin{array}{l}
0.200 \\
0.061
\end{array}\right]
$$

Looking into the estimator in Fig. 9, the dynamics of the condenser/accumulator section can never be reproduced exactly in real life. Simulation experiments were therefore run with wrong estimator dynamics by changing the $V / H$ ratio in eqn. (31). Several similar experiments showed good robustness of the combined estimation/ control scheme.

This estimation scheme is also valid for larger accumulators since the dynamics of a larger accumulator becomes slower ( $H$ increases in eqn. (31)). Hence a larger accumulator will tolerate a larger time lag between the real and estimated top plate composition $\left(x_{22}\right)$. Likewise a large accumulator will be more insensitive to a given measurement time-delay than a column with a small accumulator.

As mentioned earlier the estimation scheme presented above has to take into account the measurement noise variance when the estimation gain vector is computed, since $k_{1}$ is multiplied by the innovation process, see eqn. (30), and then fed directly back into the controller. Hence, the problem boils down to the familiar compromise between the wish for fast estimator dynamics, and the need to reduce the noise feedback to the control inputs. The problem with feedback of measurement noise may also appear in the rest of the feedback loop. In the presence of measurement noise (which have been omitted in the simulations) some sort of filtering might be necessary in order to avoid too much noise feedback to the control inputs.

The simple estimation loop in Fig. 9 is an example of a measurement noise filter. The estimate of the distillate composition is used instead of the actual measurement. The integrated product deviations, see $z_{1}$ and $z_{2}$ in eqn. (22), should however, be based on the time measurements in order to secure proper integral action.

The rather small deviations that appear in the simulation in this article require very accurate composition measurements. As discussed in $\S 2$, the non-linear model is based on a number of assumptions that will not be fulfilled in real life. Hence, the control cannot be made as tight as in the simulation experiments presented here. The demand on measurement accuracy is thereby weakened. Robustness of the proposed control strategies are essential. Subjected to any type of realistic process change, every controller must at least remain stable. It may be of particular interest to see how the reduced feedback controller, i.e. three composition measurements and a vapour flow measurement, performs at different operating points. Figures $11(a)$ and (b) (curve 1) show the response to a step change in the feed flow rate using the feedback gain matrix shown in Table 3 with the operating point obtained when the reflux/ distillate flow ratio is increased to $\mathbf{5 \cdot 0}$. The controller's performance is excellent. An extensive body of simulations at different operating points showed robustness of the reduced LQ-controller. 
(a)

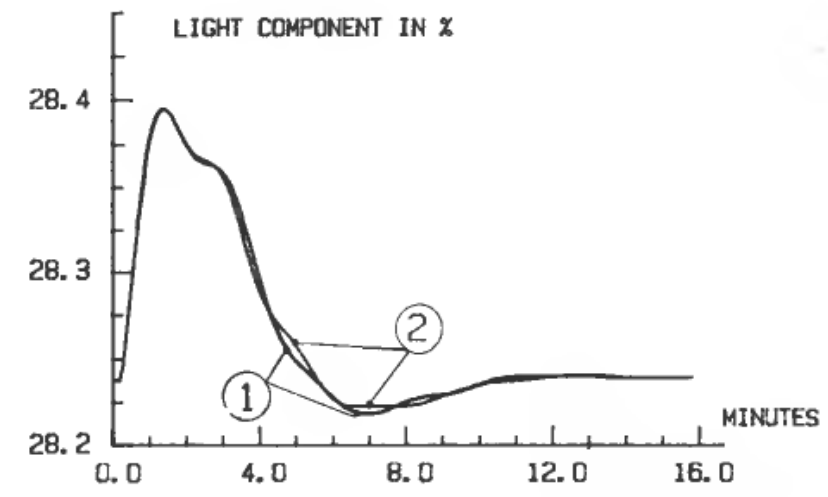

(b)

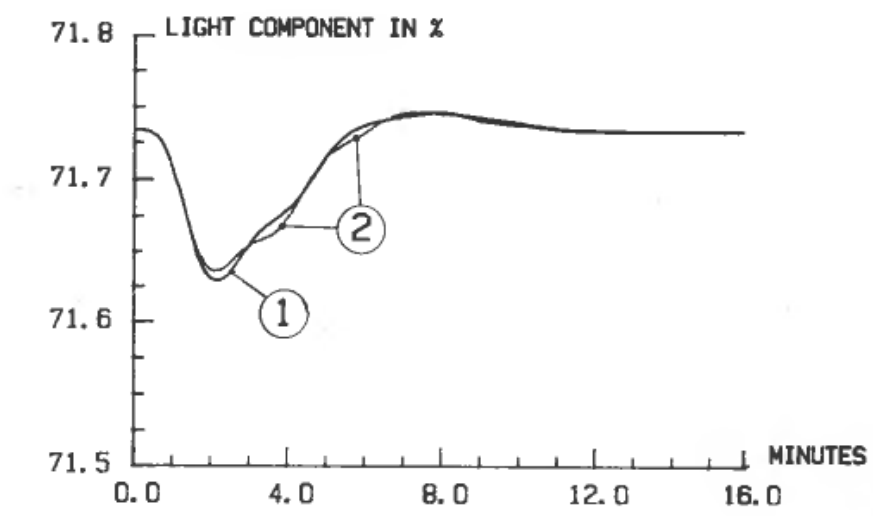

(c)

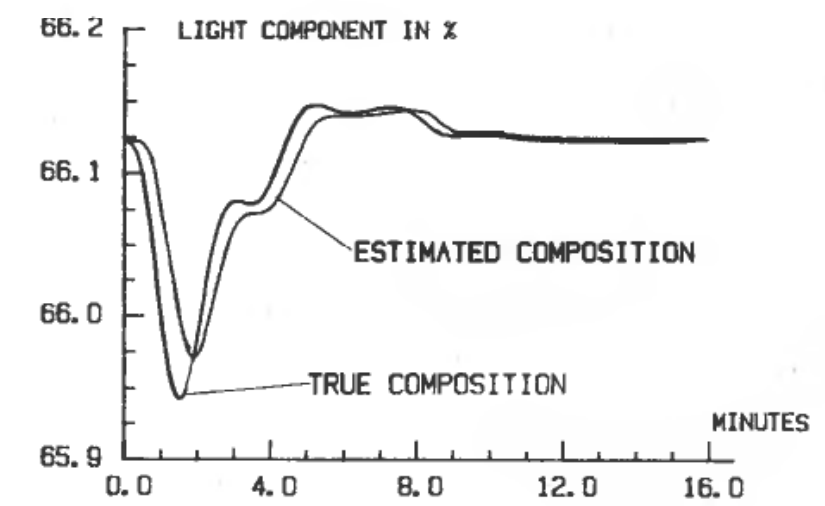

Figure 10. Responses to a step change in the feed flow rate $(a)$ and $(b)$ using a LQ-control structure with a reduced state feedback and feedforward compensation. The estimated composition (c) on the top plate is used in the control law. 
As a matter of interest the response to a step change in both the feed flow rate and the feed composition at time zero can be viewed in Figs. $11(a)$ and $(b)$ (curve 2). The controller compensates neatly for the feed upset also in this case.

This is no extensive discussion of the robustness of the proposed control structure. It does, however, indicate robustness of the controller.

(a)

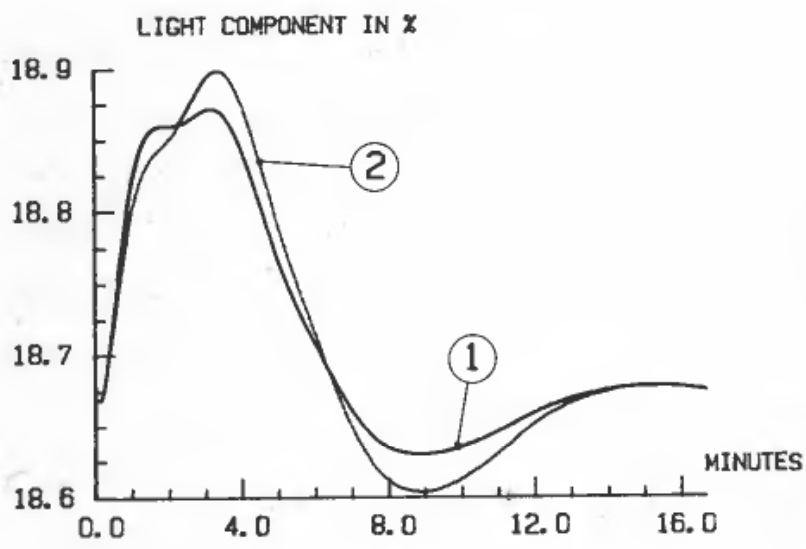

(b)

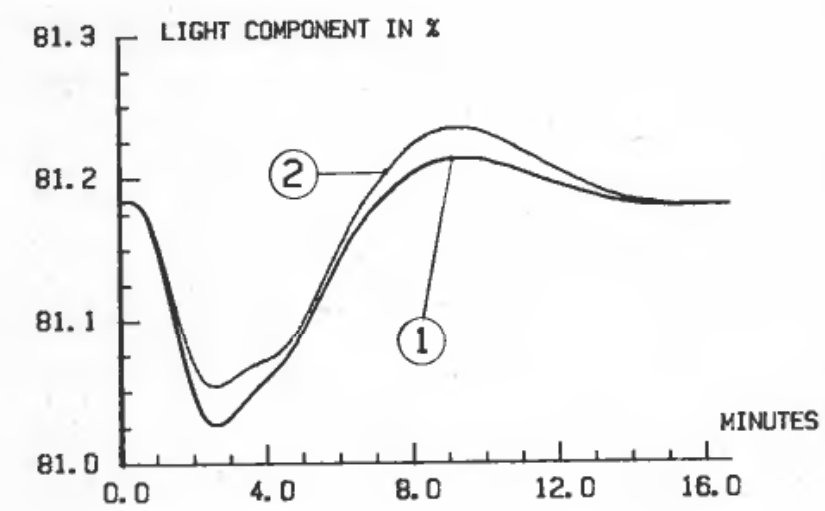

Figure 11. Responses to a step change in the feed flow rate (1) or in the feed flow rate and the feed composition (2) using a LQ-control structure with reduced state feedback and feedforward compensation for (a) bottom product composition and (b) distillate composition.

It should be emphasized that the reduced LQ-controller's complexity is comparable with the use of two PI-controllers, the amount of additional computation is small. Hence the results indicate that dual composition control can be improved with only a small increase in the controller's complexity. These results can, of course, only be verified by tests on a real column. For columns whose dominant dynamics can be described by the model used here, the hope of successful trials should be good. 


\section{Conclusion}

A fairly simple, but for this investigation quite realistic model, has been developed in order to perform control studies on a distillation column. Simulations show that a time invariant LQ-controller gives superior control compared with two well-tuned PI-controllers in a SISO-structure.

It proved quite simple to obtain weight matrices (in the LQ-criteria) that gave tight control when the structure of these matrices were based on process knowledge presuming all the dominant dynamic properties are apparent in the simplified linear model. The number of measured state variables were reduced from 24 to 4 with only a minor reduction in the control performances. The product stream compositions had to be measured in order to secure integral action. The location of the third composition measurement proved crucial. It had to be placed at the top end of the column. If not, the controller became unstable. The fourth measurement it was necessary to retain was the vapour flow measurement. The reduced state feedback scheme seemed robust subjected to operating point changes. The third measurement was omitted by introducing a simple estimation loop. Provided that the estimation loop was fairly fast, the controller's performance was hardly effected by using the estimate of the composition at the top end of the column instead of the true value.

Measurement noise was discussed suggesting that simple estimation models could serve as noise filters suppressing measurement noise.

A simple feedforward compensation from a feed flow measurement improved the control performance of both the monovariable PI-structure and the multivariable structure.

An incentive for making this investigation was to verify the results reported in Foss (1980) for larger columns than the one simulated there (seven stage column). The results obtained in this article correspond neatly with the results reported in the above mentioned reference. This indicates that the results obtained here are valid for larger columns than the one investigated in this article, if the dynamics can be reproduced fairly well by the model developed in $\S 2$. The simulations also indicate robustness of the proposed controller in the sense that it performed well at different operating points.

Only further investigations can verify the ability of the controller. Real applications will always bring out problems which have been disguised in the simulation of a physical process.

\section{ACKNOWLEDGMENTS}

The author wishes to thank Professor J. G. Balchen for inspiring and helpful discussions. Parts of this work has been financed by The Royal Norwegian Council for Scientific and Industrial Research.

\section{REFERENCES}

ECKelmanN, W. (1980). Experience with a state indicator for the control of distillation columns, Regelungstech. Prax (Germany), 22, 120-126.

Gilles, E. D., and ReTzBACH, B. (1980). Reduced models and control of distillation columns with sharp temperature profiles. IEEE Conference on Decision and Control, Alberquerque, USA, 865-870.

Hammarstrom, L. (1980). A simulation study of multivariable control and estimation strategies for chemical engineering processes, Thesis, Abo-Finland.

Hu, Y. C., and RAmireZ, W. F. (1972). Application of modern control theory to distillation columns. AIChE Journal, 18, 479-486. 
216

B. A. Toss

LeVY, R. E., Foss, A. S., and Gens, E. A. (1969). Response modes of a binary distillation column. I and Ec Fundamentals, 8, 765-776.

LOUD, G. D., and WAGgoner, R. C. (1978). The effects of interstage backmixing on the design of a multicomponent distillation column. Ind. Eng. Chem. Process Bes. Lev., 17, 149-156.

Fogs, B. A. (1980). Modelling and control of a binary distillation column (Norwegian), M.Sc. Thesis. Division of Engineering Cybernetics, Trondheim-Norway.

LUYBEN, W. L. (1979). Introduction and overview of distillation column control. FIChE Workshop, Tampa, USA, 1979.

Luyben, W. L., Tyreus, B. D., and Schiesser, W. E. (1975). Stiffness in distillation models. Ind. Eng. Chem., Process Bes. Lev., 14, 427-433.

Martin, G. D., Latour, P. R., and Richard, L. A. (1981). Closed-loop optimization of distillation energy, Chem. Eng. Prog. (USA), 77, 33-37.

Stainthorp, F. P., Searson, H. M. (1973). The dynamics of fractionating columns. Trans. Instr. Chem. Engrs, 51, 42-47. 